\title{
INFORMAÇÃO DA CONSTRUÇÃO EM USOS BIM - ESTUDO DE CASO
}

\author{
Rui Pina ${ }^{(1)}$, Miguel Chichorro Gonçalves ${ }^{(1)}$, Pedro Mêda Magalhães ${ }^{(2)}$, Diego Calvetti( ${ }^{(2)}$ \\ (1) CONSTRUCT - Gequaltec, Faculdade de Engenharia da Universidade do Porto, Porto \\ (2) CONSTRUCT - Gequaltec, Instituto da Construção, Faculdade de Engenharia da \\ Universidade do Porto, Porto
}

\begin{abstract}
Resumo
A metodologia BIM envolve processos que exigem uma elevada colaboração e interoperabilidade entre todos os intervenientes na construção, devido à partilha de um vasto conjunto de informação, gráfica e não gráfica, que deve ser adequadamente utilizada de modo a otimizar o processo construtivo.

A organização e uniformização da informação constitui assim um elemento chave para a implementação plena de metodologias de trabalho BIM, permitindo impulsionar um ambiente de mais efetiva colaboração entre os intervenientes na construção, bem como uma maior eficiência tanto dos processos como das realizações.

O presente trabalho tem como base o documento "Análise e Contextualização da Informação Técnica na Construção - Estudo de Caso", [1], e explora a informação técnica existente na construção em vários níveis de detalhe, tendo-se para o efeito abordado um caso de estudo referente a um edifício existente, para o qual foi considerado um conjunto restrito de elementos construtivos. Numa primeira fase procurou-se, para cada um desses elementos, o enquadramento num sistema de classificação personalizado, baseado em sistemas de classificação nacionais existentes, com o objetivo de analisar e perceber qual a maneira mais eficiente de organizar esta informação num contexto BIM. Numa segunda fase foi recolhida informação, de forma ampla e exaustiva referente a cada um desses elementos, de forma a poder posteriormente efetuar a sua contextualização em vários usos BIM, isto é, inserir e compartimentar essa informação em vários contextos de aplicação na construção, sendo esta fase de maior incidência no presente trabalho.

O enquadramento da informação técnica da construção nas diferentes áreas e fases do processo construtivo permite um fluxo de informação mais focalizado para aquilo que é primordial em cada fase, separando assim aquilo que é a informação útil e informação dispensável, evitando assim excessos de informação.

Este trabalho tem o objetivo de analisar os efeitos da separação da informação em vários domínios da construção, testando um modelo organizacional personalizado que cria outputs de informação para diversos contextos da construção. Para além disso é também um propósito do presente trabalho averiguar a ambiguidade de informação em diferentes contextos da construção.
\end{abstract}




\section{Introduçãa}

O desenvolvimento tecnológico verificado nas últimas décadas influencia toda a indústria e pode dar as ferramentas necessárias para implementar a interoperabilidade no setor da construção civil. De entre as várias teorias e métodos baseados no uso de softwares na construção, as metodologias BIM têm ganho relevância nos últimos anos e promete revolucionar o mercado da construção. As metodologias de trabalho BIM visam a criação de um ambiente mais colaborativo e comum às várias entidades envolvidas no processo construtivo, de modo a que haja eficiência da transmissão da informação através das várias etapas, reduzindo assim o número de desperdícios e perdas de informação, que acarretam tempo e custos adicionais. $\mathrm{O}$ uso do BIM permite uma maior qualidade na fase de projeto, através da criação de modelos de objetos ricos em informação, mas também potencialidades que abarcam todo o processo construtivo até à fase de utilização.

O BIM implica consequentemente uma elevada complexidade ao nível de informação, tanto ao nível de detalhe, como ao nível da sua organização, e a sua articulação ao longo do processo construtivo constitui assim um enorme desafio._A organização da informação constitui uma das dificuldades a nível nacional pela falta de um sistema explícito de classificação da informação na construção, bem como a falta de bibliotecas de informação devidamente especificadas.

O objetivo deste trabalho é o de avaliar a forma como se organiza a informação na construção, procurando perceber de que modo é feita a distribuição dessa informação, tendo em conta as diversas fases e áreas de intervenção do processo construtivo, e que outputs podem obter a partir da manipulação da informação, de modo a poder dar apoio ao processo construtivo e colmatar as ineficácias que lhe estão presentemente associadas nas diversas fases da construção. A prova do conceito neste artigo é feita na aplicação ao elemento construtivo porta exterior dentro do trabalho mais abrangente de Pina, [1] para 10 elementos construtivos.

\section{Sistemas de classificação da informação da construção}

\subsection{Contextualização da Informação técnica}

Um sistema de classificação da informação funciona como uma gestão do conhecimento, que visa ordenar os dados, independentemente do seu tipo, em catalogações adequadas. Estes sistemas devem ser estáveis e flexíveis, de modo a manter a sua estrutura consoante o contexto de aplicação e a adição de novos elementos.

No setor da construção, a ISO (International Organization for Standardization) tem produzido normas com o objetivo de uniformizar a classificação e troca de informação, como é o caso da (ISO 12006 e ISO19650, [2,3]), cujo objetivo passa por orientar a estruturação de sistemas de classificação. Com efeito, um sistema concebido de acordo com a ISO12006, [2] deve abranger todo o ciclo de vida do empreendimento, contemplar grande variedade de trabalhos de construção, incluindo instruções, conceção, documentação, construção, operação, manutenção e demolição, [4]. A importância da correta contextualização da informação técnica no ambiente da Indústria da Construção (IC) durante todo o ciclo de vida construtivo potencia benefícios vários para os donos de obra, projetistas, construtores, clientes, fiscalização, cadeias de fornecimento e todos os envolvidos. Os processos desenvolvidos no artigo, baseados em conceitos e princípios normativos implementam oportunidades, redução de riscos e de custos na produção e utilização dos bens produzidos na IC. Novos ambientes de colaboração entre 
todos os participantes nos projetos da IC e na sua gestão são fundamentais para a eficiência na entrega e operação dos bens construídos. Pelo que a informação técnica tem que ser contextualizada e criada uma estrutura de gestão dessa informação.

\subsection{Organização dos sistemas de classificação}

Os sistemas de classificação da informação da construção geralmente agrupam os objetos conforme um de dois modelos organizacionais, adotando uma classificação hierárquicanumerativa ou analítica-sintética.

Uma classificação hierárquica-numerativa segue uma estrutura hierárquica rígida e unidimensional. Os elementos estão agrupados em conjuntos e subconjuntos, sendo que cada elemento é classificado numa única categoria. Numa classificação analítica-sintética, pelo contrário, o objeto não é incluído num conjunto concreto, sendo descrito simultaneamente por várias características, sendo assim um sistema mais flexível a modificações, e por isso mais usado nos padrões BIM.

\subsection{Sistemas de classificação existentes}

Abordando em primeiro lugar sistemas de classificação internacionais, constata-se já um nível organizacional sólido em algumas regiões, que têm sistemas de classificação já compatibilizados com padrões de trabalho BIM. Alguns dos principais sistemas de classificação encontram-se dispostos no Quadro 1.

Quadro 1: Sistemas internacionais de classificação

\begin{tabular}{lcc}
\hline Sistema de Classificação & Região de Origem & Tipo de Classificação \\
\hline Uniclass 2015 & Reino Unido & Analítica-sintética \\
SfB & Suécia & Analítica-sintética \\
OmniClass & EUA & Analítica-sintética \\
UniFormat & EUA e Canadá & Hierárquica-enumerativa \\
MasterFormat & EUA e Canadá & Hierárquica \\
Natspec & Austrália & Analítica-sintética \\
\hline
\end{tabular}

A nível nacional o paradigma é diferente, não existindo ainda um sistema explícito para a classificação da informação na construção. Existem sistemas baseados em análises estatísticas (Quadro 2), entre outros, sendo do ProNIC o sistema mais adaptado para a gestão da informação na construção. Estes sistemas seguem, regra geral, um modelo hierárquico, que consequentemente carecem de flexibilidade no apoio a metodologias de trabalho BIM. 
Quadro 2: Sistemas de classificação nacionais e respetivas entidades responsáveis

\begin{tabular}{|c|c|}
\hline Sistema de classificação & Entidade Responsável \\
\hline $\begin{array}{l}\text { Classificação Portuguesa das Atividades Económicas } \\
\text { (CAE-VER.3) }\end{array}$ & Instituto Nacional de Estatística \\
\hline Vocabulário Comum dos Contratos Públicos (CPV) & Comissão Europeia \\
\hline Classificação Portuguesa das Construções (CC-PT) & Instituto Nacional de Estatística \\
\hline $\begin{array}{l}\text { Protocolo para a Normalização da Informação Técnica } \\
\text { na Construção (ProNIC) ®), [5] }\end{array}$ & $\begin{array}{l}\text { Instituto dos Mercados Públicos, } \\
\text { do Mobiliário e da Construção }\end{array}$ \\
\hline
\end{tabular}

\subsection{Propriedades de objetos}

Um sistema de classificação da informação na construção deve apresentar em última instância as propriedades relativas a cada objeto. Estas propriedades são fundamentais nas várias tomadas de decisão durante o processo construtivo, já que são estas mesmas propriedades que definem o elemento em si.

Num sistema de classificação hierárquico, atingindo um nível de detalhe em que se pode isolar um objeto, obteremos um conjunto extenso e exaustivo de propriedades, sem ligação e articulação à finalidade de aplicação dessa informação.

\section{Usos BIM}

\subsection{Introdução}

Um uso BIM define-se como uma área, entidade ou função no processo construtivo, alvo da aplicação de metodologias BIM. Foram definidos para a análise deste caso de estudo os seguintes usos:

- Promoção e investimento imobiliário;

- Elaboração de projetos;

- Execução da obra;

- Gestão de ativos.

O enquadramento e contextualização da informação técnica nos usos acima mencionados será feita tendo em conta o tipo de empreendimento abordado no caso de estudo e depende naturalmente dos materiais e produtos considerados, da sua maior ou menor quantidade e variedade de informação.

\subsection{Promoção e investimento imobiliário}

Pode considerar-se a promoção como uma valorização de todos os produtos, materiais e equipamentos utilizados na construção, de modo a satisfazerem as necessidades propostas aquando da construção do empreendimento. A metodologia BIM apresenta qualidades que permitem potenciar a promoção a começar pela criação de modelos 3D que permitem uma visão muito aproximada daquilo que vem a ser o produto final e toda a informação relevante 
envolvida. A metodologia também facilita a exploração de alternativas devido ao menor esforço para a representação do modelo quando comparada com outras metodologias.

\subsection{Elaboração de projetos}

A envolvência de metodologias BIM ao nível do projeto apresenta competências já credenciadas. As maiores potencialidades, comparativamente a métodos mais tradicionais como softwares CAD, refletem-se numa melhor coordenação do projeto, estimativa de custos mais precisa e antecipação de potenciais erros em fases mais avançadas. As metodologias BIM têm as ferramentas necessárias para uma melhor qualidade na elaboração do projeto. $\mathrm{O}$ uso destas metodologias acaba por aumentar o tempo de dedicação ao projeto, bem como o custo da sua elaboração, porém as vantagens acabam por ser alcançadas em fases posteriores. A documentação de projeto é uma parte que envolve grandes quantidades de informação, entendendo-se que uma biblioteca de informação associada aos materiais e produtos de construção exclusiva para esta fase pode ter um impacto positivo na produtividade, visto eliminar informação desnecessária e clarificar as necessidades do projeto.

\subsection{Execução da obra}

A fase de execução da obra é uma fase onde ocorrem conflitos por vezes inesperados, que se podem refletir em aumentos de custos e prazos, afetando a produtividade. Estes conflitos podem ter diferentes origens ao nível da fase de construção, tais como o deficiente planeamento da obra, atrasos por parte dos fornecedores e qualidade imprópria dos materiais empregues (Cabrita, 2008). Segundo um estudo desenvolvido em 2009 (Pilar et al., 2009), as principais causas de atrasos na construção são, por ordem de ocorrência as seguintes:

- Alterações ao projeto inicial;

- Atrasos nas aprovações de fiscalização;

- Erros de planeamento e avalização do projeto;

- Condições climatéricas;

- Falta de mão de obra especializada;

- Falta de material;

- Falta de comunicação interna;

- Falta de equipamento;

- Falta de mão de obra especializada;

- Falha na segurança e ocorrência de acidentes.

Apesar de nem todas estas causas derivarem do processo da construção em si, entende-se que a qualidade e organização da informação adotada para a preparação e no decorrer da obra pode travar ou atenuar alguns desses conflitos.

\subsection{Gestão de Ativos}

A área da gestão de ativos, associada ao termo inglês facility management, apresenta uma elevada relevância na fase de utilização de um edifício, e corresponde a um conjunto de boas práticas que objetivam a otimização dos ativos num dado empreendimento. Essas práticas, sendo corretamente aplicadas, permitem obter várias vantagens (Sousa, 2016), nomeadamente redução de custos de manutenção, gestão de riscos, melhor controlo das instalações, maior segurança e maior consciência do desempenho da organização. 
Estas vantagens podem ser potenciadas pela adoção de metodologias BIM. O facto de toda a informação necessária à gestão do empreendimento poder existir num só modelo facilita o seu controlo, e a quantidade da informação introduzida no modelo pode ser medida de modo a satisfazer os vários interesses ao nível da gestão. Esta área representa a sétima dimensão do BIM, e permite, para além de outras utilidades, o desenvolvimento de estratégias do ciclo de vida do empreendimento, planos de manutenção e planos de suporte técnico. Para isso, é necessário um tipo de informação que se adeque a essas funcionalidades.

\section{Contextualização da informação em usos BIM - Descrição da metodologia}

A metodologia adotada neste trabalho, esquematizada na Figura 1, aborda a informação técnica existente na construção, devidamente classificada e organizada por níveis de decrescente grau de complexidade, de um modo hierárquico, baseado em sistemas de classificação nacionais. $\mathrm{O}$ último nível de informação contém os parâmetros que definem cada objeto que são, no seguimento da metodologia, desagregados e inseridos em diferentes contextos, denominados usos BIM. Foram selecionados para o presente caso de estudo quatro domínios de aplicação dentro do processo construtivo, que correspondem aos designados usos BIM: promoção e investimento imobiliário, elaboração de projeto, execução da obra e gestão de ativos.

Tendo como base o caso de estudo utilizado por Pina em "Análise e Contextualização da Informação Técnica na Construção em Usos BIM - Estudo de Caso", [1], foi efetuada uma listagem dos sistemas, materiais e equipamentos. Esta informação obtida, devido à sua variada complexidade foi organizada e classificada. Para o efeito, procedeu-se a uma hierarquização dessa informação, distribuída por vários níveis de detalhe, até chegar a um nível em que se encontram apenas os produtos e materiais de construção, que constituem assim uma amostra de elementos. A esses elementos, que já podemos denominar objetos foi seguidamente associado um conjunto de propriedades, que correspondem à informação recolhida através da análise de normas, sistemas de classificação de informação, fichas técnicas e outras fontes, constituindo assim uma base de dados ampla e variada para dar suporte à fase que se segue, de contextualização dessas propriedades nos usos BIM adotados.

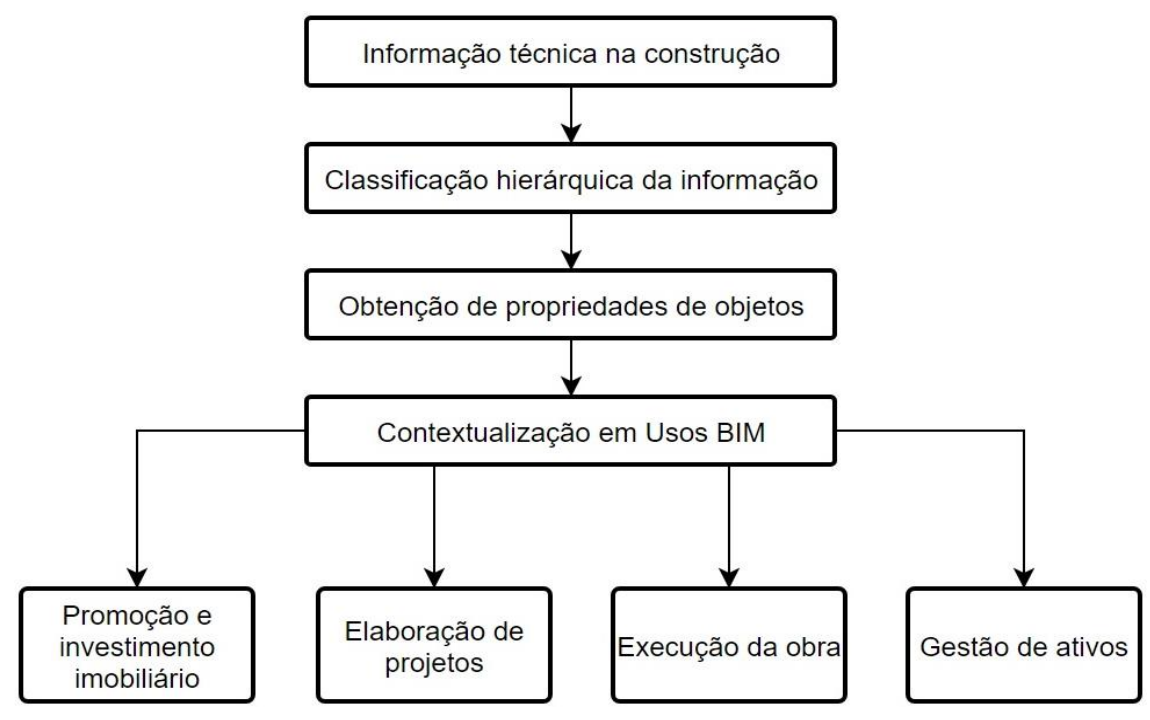

Figura 1: Esquema da metodologia abordada. 
$\mathrm{Na}$ última fase deste método foi feita a dita contextualização, isto é, uma desagregação e distribuição das propriedades/parâmetros associados a cada objeto pelos usos BIM referenciados, onde se avaliou a importância da informação relativa a produtos e materiais de construção em cada uso. Este cruzamento de informação pode ser esquematizado através de uma matriz, como exemplificado na Figura 2, e consistiu numa análise subjetiva, crítica e justificativa das escolhas.

\begin{tabular}{lcccc}
\hline & $\begin{array}{c}\text { Promoção e } \\
\text { investimento } \\
\text { imobiliário }\end{array}$ & $\begin{array}{c}\text { Elaboração de } \\
\text { projetos }\end{array}$ & $\begin{array}{c}\text { Execução de } \\
\text { obra }\end{array}$ & Gestão de ativos \\
\hline Propriedade 1 & $\mathrm{X}$ & $\mathrm{X}$ & $\mathrm{X}$ & $\mathrm{X}$ \\
Propriedade 2 & $\mathrm{X}$ & - & $\mathrm{X}$ & $\mathrm{X}$ \\
Propriedade 3 & $\mathrm{X}$ & $\mathrm{X}$ & $\mathrm{X}$ & $\mathrm{X}$ \\
Propriedade 4 & $\mathrm{X}$ & - & $\mathrm{X}$ & - \\
\hline
\end{tabular}

Figura 2: Matriz tipo propriedades versus usos BIM.

Para aplicação da metodologia irá ser analisado um objeto específico e um conjunto de propriedades limitado. Para o efeito selecionou-se, de entre os materiais e produtos analisados por Pina [1], o caso da porta exterior, já que constitui um elemento facetado em termos de propriedades e áreas de aplicação na construção, pelo que constitui um elemento adequado à aplicação da metodologia.

\section{Classificação dos produtos e propriedades}

\subsection{Níveis de classificação}

A informação presente no caso de estudo foi, conforme a metodologia descrita, organizada e classificada por níveis com um grau crescente de particularidade, começando por grupos de disposições construtivas, seguindo-se de subgrupos de disposições construtivas, até chegar aos materiais de construção (Quadro 3). Por fim, as propriedades intrínsecas aos materiais de construção.

Os níveis da hierarquia de classificação adotada são baseados na classificação utilizada no livro: Curso sobre Regras de Medição na Construção [9], semelhante à classificação utilizada no ProNIC®, [5].

Quadro 3: Os 3 primeiros níveis de classificação do produto analisado

\begin{tabular}{cccccc}
\hline & $\mathbf{1}^{\mathbf{0}}$ Nível & \multicolumn{2}{c}{$\mathbf{2}^{\mathbf{0}}$ Nível } & \multicolumn{2}{c}{$\mathbf{3}^{\mathbf{0}}$ Nível } \\
\hline Grupo & Designação & Subgrupo & Designação & Material & Designação \\
\hline 15. & $\begin{array}{c}\text { Elementos de } \\
\text { serralharia }\end{array}$ & 15.1 & $\begin{array}{c}\text { Componentes } \\
\text { em vãos }\end{array}$ & 15.1 .1 & $\begin{array}{c}\text { Porta } \\
\text { exterior }\end{array}$ \\
\hline
\end{tabular}


O último e mais detalhado nível de informação adotado corresponde às propriedades dos produtos, e é onde se deve dar maior foco para a compreensão da metodologia adotada. Tratase de um nível de informação particular a cada produto, que é ainda agrupada em categorias de parâmetros, como se observa no Quadro 4. Estas propriedades encontram-se codificadas e agrupadas por categorias. Na coluna relativa ao parâmetro encontra-se a designação dos parâmetros adotados, seguidas das unidades, quando aplicáveis ao parâmetro em causa. A última coluna descreve a origem e fundamento desse parâmetro, devidamente especificados nos Quadros 4 e 5.

Quadro 4: Categoria de características físicas e geométricas da porta exterior

\begin{tabular}{lcccc}
\hline $\mathbf{N}^{\mathbf{0}}$ & Categoria & Parâmetro & Unidades & Origem \\
\hline 15.1.1.8. & & Material & Texto & Ss_25_30_20_30 \\
15.1.1.9. & & Dimensões & N/A & 49-71 19 00 \\
15.1.1.9.1. & Altura & $\mathrm{mm}$ & NP EN 14351-1 \\
15.1.1.9.2. & Características & Comprimento & $\mathrm{mm}$ & $49-711913$ \\
15.1.1.9.3. & físicas e & Espessura & $\mathrm{mm}$ & $49-711925$ \\
15.1.1.10. & geométricas & Peso por unidade & $\mathrm{kg}$ & $49-716913$ \\
15.1.1.11. & & Cor & Texto & $49-614153$ \\
15.1.1.12. & & Acabamento & Texto & $49-614157$ \\
15.1.1.13. & & Tipo de abertura & Texto & $49-81613511$ \\
\hline
\end{tabular}

As propriedades relacionadas com cada objeto foram obtidas na sua maioria através da análise de vários elementos e documentos de cinco seguintes fontes de informação: as Normas Portuguesas, (NP), que também incluem as homologadas pelas normas europeias (NP EN), as normas harmonizadas da união europeia $(E N)$ e os sistemas de classificação internacionais OmniClass e UniClass 2015. Foi também utilizada como fonte o manual de condições técnicas do ProNIC, e para além destas fontes, foi obtida informação através da consulta de fichas técnicas e outros documentos dos diversos produtos.

Quadro 5: Fontes dos parâmetros dos produtos

\begin{tabular}{lc}
\hline Designação & Nomenclatura (Exemplo) \\
\hline Norma Portuguesa & NP 100 ou NP EN 100 \\
Norma Europeia & EN 100 ou EN 100-1 \\
ProNIC & ProNIC \\
OmniClass & $49-11$ 11 11 \\
UniClass 2015 & Pr_10_10_10_10 ou Ss_10_10_10_10 \\
\hline
\end{tabular}


Fez-se uma breve abordagem aos sistemas de classificação OmniClass e UniClass 2015 por ter representatividade internacional. O OmniClass é um sistema de classificação americano especializado para a construção com várias aplicações entre as quais organização de bibliotecas de materiais, especificações de produtos e informações de projetos, e segue o quadro internacional estabelecido pela ISO. A OmniClass conta atualmente com dezassete tabelas de informação variada, sendo para este caso utilizada a tabela 49, referente às propriedades de variados produtos da construção, que se encontram devidamente organizadas e definidas. Ainda o sistema UniClass 2015 que conta com um conjunto de quadros com especificações acerca das propriedades dos produtos, e apresenta um nível de compatibilidade com plataformas de trabalho BIM.

\section{Análise crítica e contextualização da informação nos usos BIM}

A informação na construção tem uma amplitude e diversidade elevada, e num ambiente de trabalho BIM, onde é vital o desenvolvimento de modelos ricos e regrados no que toca à informação, pode por vezes existir um congestionamento e excesso de informação por vezes desnecessária em determinadas fases do projeto.

\subsection{Análise dos parâmetros}

A informação dos parâmetros a analisar encontra-se disposta no Quadro 4. A análise dos parâmetros englobados nas características gerais, características físicas e geométricas, o desempenho e as condições de distribuição e armazenamento e propriedades é feita minuciosamente por Pina (Pina 2019). No presente documento irá ser feito apenas o enquadramento da informação contida no Quadro 4, que corresponde a uma categoria de parâmetros relativos ao objeto.

\subsection{Enquadramento nos usos BIM}

Devido à elevada diversidade de informação presente na construção, e no seguimento da metodologia adotada pretende-se agora uma contextualização, isto é, uma compartimentação da informação que se considera adequada para cada uso BIM enunciado.

Defende-se nesta fase do método uma separação da informação, de modo a poder atribuir a informação necessária a cada fase, retirando desta forma a informação desnecessária para cada fase, permitindo um ambiente de trabalho otimizado e totalmente focalizado para cada uso.

\subsection{Exemplo da porta exterior}

A contextualização da informação para o produto aqui analisado encontra-se materializado no Quadro 6, com a informação proveniente do Quadro 5, e seguindo a lógica de cruzamento de informação espelhada na matriz da Figura 2. 
Quadro 6: Distribuição das propriedades nos usos BIM da porta exterior

\begin{tabular}{|c|c|c|c|c|}
\hline \multirow[b]{2}{*}{$\begin{array}{l}\text { Código da } \\
\text { propriedade }\end{array}$} & $\begin{array}{c}\text { Promoção e } \\
\text { investimento } \\
\text { imobiliário }\end{array}$ & $\begin{array}{c}\text { Elaboração de } \\
\text { projetos }\end{array}$ & $\begin{array}{c}\text { Execução da } \\
\text { obra }\end{array}$ & Gestão de ativos \\
\hline & & 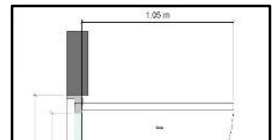 & 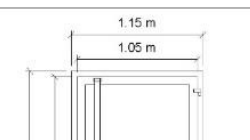 & \\
\hline 15.1.1.8. & $X$ & $X$ & & \\
\hline 15.1.1.9. & & $X$ & $X$ & \\
\hline 15.1.1.9.1. & & $\mathrm{X}$ & $X$ & \\
\hline 15.1.1.9.2. & & $\mathrm{X}$ & $X$ & \\
\hline 15.1.1.9.3. & & $X$ & $\mathrm{X}$ & \\
\hline 15.1.1.10. & & & $X$ & \\
\hline 15.1.1.11. & $X$ & & & \\
\hline 15.1.1.12. & $\mathrm{X}$ & & & \\
\hline 15.1.1.13. & & & & $X$ \\
\hline
\end{tabular}

As questões estéticas, como a cor e o acabamento têm importância, no que toca à promoção, bem como o material. Para além das propriedades descritas no Quadro 4, considera-se ainda importante para este uso BIM o fabricante, o preço e a durabilidade.

No ponto de vista do projeto, como se verifica no Quadro 6, são necessárias as propriedades que permitam, como é evidenciado na figura, fazer uma correta representação espacial da porta. Para além dessas propriedades geométricas, o material que compõe o elemento também deve estar já evidenciado no projeto.

Além dessas, são igualmente importantes as características de desempenho, estas não representadas no Quadro 4, onde entram propriedades relacionadas com a questões térmicas, luminosas e de permeabilidade ao ar para a efetuação de cálculos relativos a essas mesmas especialidades. Neste caso, também se considera importante para a elaboração da documentação a resistência ao impacto, que deve ser adaptada ao uso que a porta poderá vir a ter.

$\mathrm{Na}$ gestão de ativos incluem-se, dentro das propriedades descritas no Quadro 4, o tipo de abertura, já que tem influência na gestão da sua manutenção. Para além destas, as informações do fornecedor, informações relativas ao preço de reposição, data de construção, tempo de vida expectável, método de limpeza e informações de garantia devem ser evidenciadas. 


\section{Conclusões}

O desenvolvimento e aplicação do método utilizado no presente trabalho permitiu alcançar algumas conclusões e vincar algumas premissas inicialmente levantadas. Em primeiro lugar, conclui-se a existência de uma vasta diversidade de informação na construção, com limites por vezes não explorados no que toca a determinados parâmetros e níveis de detalhe. Nota-se claramente a inexistência de um sistema de classificação da informação organizado e especializado para a área da construção. O ProNIC apresenta-se como uma plataforma que possui grandes potencialidades no que toca à criação de documentação na construção, e apresenta também uma classificação da informação com vários níveis, já especificados.

No entanto, sente-se que é fundamental para todo o panorama nacional ao nível da construção, a existência de uma biblioteca unicamente destinada à especificação da informação na construção, que sirva de base para a implementação de metodologias de trabalho BIM. A existência desta biblioteca permitiria assim um maior nível de uniformização da informação, fomentando também um ambiente mais colaborativo. Em países mais desenvolvidos no que toca a utilização de metodologias BIM verifica-se já a existência desse tipo de bibliotecas, como por exemplo a UniClass 2015 no Reino Unido.

Verifica-se também alguma dificuldade em conseguir encontrar e reunir informação de produtos e materiais de construção. A nível nacional, apenas foi possível encontrar informação a partir de normas harmonizadas e informação retirada através do ProNIC.

A contextualização da informação neste trabalho foi feita com base numa análise crítica e subjetiva, porém fundamentada, e permitiu chegar a algumas conclusões seguidamente especificadas:

- Existe informação necessária e, pelo contrário, outra que é supérflua em certas áreas e fases do processo construtivo, podendo ser dispensada;

- A orientação da informação na construção para os diferentes usos facilita o processo de trabalho e as relações entre vários intervenientes do processo construtivo, por proporcionar uma linguagem adaptada a cada especialidade.

Devido ao esforço reduzido para representação e alteração de modelos de informação que os BIM permitem, é possível a ramificação e criação de vários fluxos de informação, que devem ser manipulados para o uso pretendido dentro do processo construtivo, que serão irrefutavelmente potenciados pela criação de bibliotecas de objetos organizadas e devidamente classificadas para produtos e materiais de construção no panorama nacional e associá-los aos usos BIM.

\section{Referências}

[1] R. Pina, 'Análise e contextualização da informação técnica na construção em usos BIM Estudo de caso', Dissertação do Mestrado em Engenharia Civil - Especialização em Construções, Faculdade de Engenharia da Universidade do Porto, 2019.

[2] ISO 12006-2:2015, Building construction - Organization of information about construction works - Part 2: Framework for classification, 2015. 
[3] BS EN ISO 19650-1:2018, Organization anda digitalization of information about buildings and civil Engineering works, including building information modelling (BIM) Information management using building information modelling - Part 1: Concepts and principles, 2018.

[4] H. Nunes, 'Sistemas de classificação da informação na construção', Universidade Nova de Lisboa, 2016.

[5] PRONIC, Consórcio - Resumo Executivo - Funcionalidades URL: http://www.impic.pt/impic/assets/misc/pdf/documentos_de_iniciativas_estrategicas/proni c.pdf>, 2015.

[6] A. Cabrita, 'Atrasos na Construção: Causas, Efeitos e Medidas de Mitigação.', Instituto Superior Técnico, 2008.

[7] F. Pilar et al., 'Principais causas de atrasos do plano de trabalho de uma obra de construção civil', In Engenharia 2009 - Inovação e Desenvolvimento. Covilhã: 2009.

[8] A. Sousa, 'Aplicação da Metodologia BIM-FM a um caso prático', Instituto Superior de Engenharia do Porto, 2016.

[9] M. Santos Fonseca, 'Regras de Medição na Construção', Laboratório Nacional de Engenharia Civil. ISBN 972-49-1739-8, 2000. 